\title{
Dynamic Slow Motion Video Endoscopy as an Adjunct to Impedance Audiometry in the Assessment of Eustachian Tube Function
}

\author{
Sanoop E. Sanu ${ }^{1}$ Shilpa Divakaran ${ }^{2}$ Sabarinath Vijayakumar ${ }^{3} \quad$ Sunil Kumar Saxena $^{1} \quad$ Arun Alexander $^{1}$ \\ Suryanarayanan Gopalakrishnan ${ }^{1}$
}

${ }^{1}$ Department of ENT, Jawaharlal Institute of Post Graduate Medical Education, Puducherry, India

${ }^{2}$ Department of ENT, Pondicherry Institute of Medical Sciences, Puducherry, India

${ }^{3}$ Department of ENT, Sri Manakula Vinayagar Medical College and Hospital, Puducherry, India

Address for correspondence Dr. Arun Alexander, MS, Head of the ENT Department, Department of ENT, JIPMER, Dhanvantri Naga, Gorimedu, Puducherry, India - 605006 (e-mail: shils.diva@gmail.com).

Int Arch Otorhinolaryngol 2018;22:141-145.

\begin{abstract}
Keywords

- tympanometry

- Eustachian tube function

- dynamic slow motion video endoscopy

Introduction Eustachian tube (ET) dysfunction plays an important role not only in the pathophysiology of various middle ear disorders, but also in predicting the outcome of the treatment. As there is no single test that assesses both the anatomic and physiological functions of the ET, a combination of tympanometry and dynamic slow motion video endoscopy may improve the sensitivity of ET function assessment. Objective To find out if there is any correlation between dynamic slow motion nasal video endoscopy and impedance audiometry in assessing ET function in patients with middle ear diseases.

Methods Ours was a descriptive study performed with 106 patients attending the Ear, Nose and Throat (ENT) Outpatient Department of a tertiary care center in South India with features suggestive of middle ear disease. All patients underwent impedance audiometry and dynamic slow motion nasal video endoscopy, and were graded based on the severity of the ET pathology.

Results A total of 47 out of 97 patients with abnormal endoscopy findings also had abnormal impedance audiometry. The correlation was greater among the patients with higher grades of ET dysfunction. The endoscopy findings of 106 cases, when correlated with middle ear manometry, revealed that 56 cases showed complete agreement, and 50 cases showed disagreement. The nasal endoscopy results, when correlated with middle ear manometry studies by using McNemar's chi-squared $\left(x^{2}\right)$ test, showed a significant association between the 2 tests $(p=0.017)$.

Conclusion There is a significant alteration in middle ear pressure as the severity of the ET tube dysfunction increases. Impedance audiometry and nasal endoscopy provide a better measure of ET function.
\end{abstract}

\section{Introduction}

Eustachian tube (ET) dysfunction is considered the primary pathology in most middle ear diseases. An anatomically patent
ET with normal physiological functioning is essential for the regulation of middle ear pressure and for the clearance of middle ear secretions into the nasopharynx. A disturbance in received

December 12, 2016

accepted

April 10, 2017

published online

June 16, 2017
DOI https://doi.org/

10.1055/s-0037-1603920.

ISSN $1809-9777$.
Copyright $(2018$ by Thieme Revinter

Publicações Ltda, Rio de Janeiro, Brazil
License terms

(c) (i) $\ominus$ (\$) 
these functions can lead to various diseases, like serous otitis media, adhesive otitis media, and acute or chronic suppurative otitis media (CSOM). ${ }^{1}$ The assessment of tubal function has an important role while planning the management of middle ear diseases. The status of the tubal function is a good predictor of the surgical outcomes following tympanoplasty procedures. ${ }^{2}$ It is always advisable to detect and manage the treatable causes of ET dysfunction before proceeding to the surgical management of middle ear diseases.

Several techniques have been developed recently to assess tubal function. Advances in radiologic imaging and the popularization of fiber optic endoscopes have led to a more detailed understanding of the structure and function of the ET. However, it continues to be challenging for the Otologist to differentiate between the anatomic and physiological dysfunctions of the ET despite the development of various tubal function tests; a single technique for the assessment of both anatomic and physiological functioning is yet to be developed. ${ }^{3}$

Impedance audiometry is the most widely used method to assess the middle ear pressure regulation of the ET in patients with middle ear diseases. Even though tympanometry is an objective test, high variability has been found in the results among different tympanometry-based ET function tests. It can often miss subtle anatomical abnormalities that cause functional deficit. ${ }^{3}$

Endoscopic examination of the pharyngeal orifice of the ET is quite helpful to note the occurrence of edema, inflammation, discharge, polyps and obstruction by a hypertrophied adenoid or a nasopharyngeal mass in patients with middle ear diseases. Inadequate or uncoordinated movements of the tubal orifice associated with conditions like cleft palate can be visualized using a rigid nasal endoscope. ${ }^{4}$

This study attempts to assess the correlation between dynamic nasal video endoscopy of the tubal orifice and tympanometry in assessing ET function in patients with middle ear diseases.

\section{Materials and Methods}

This descriptive study was conducted in a tertiary care center in South India. The study protocol was reviewed and approved by the Institute's Ethical Committee. Patients aged between 15 to 65 years attending the Ear, Nose and Throat (ENT) Outpatient Department over a period of 18 months with history and clinical findings suggestive of middle ear disease were included in the study. The procedures were explained to all the patients, and written informed consent was obtained from them. Patients with nasal/nasopharyngeal masses and/ or active sinonasal or middle ear infections were excluded from the study. A total of 106 patients who fulfilled the inclusion criteria were selected. Only one ear was selected from each patient. The size of the sample was estimated with an expected rate of agreement of $60 \%$ between the procedures at $5 \%$ level of significance and $90 \%$ power. $^{5}$

A detailed history was taken, and a careful ENT examination was performed, including an examination under the microscope. All patients underwent impedance measurements using a Madsen Zodiac 901 machine (Otometrics, Denmark), which was calibrated according to the American National Standards Institute (ANSI) 1969 standards. The admittance tympanogram was recorded for the patients with intact tympanic membranes using a $226-\mathrm{Hz}$ probe tone. The parameters recorded were static admittance, peak pressure, and ear canal volume to record a tympanogram. The starting pressure was +200 decapascals (daPa); the pressure varied from +200 daPa to -400 daPa; the pump speed was 200 daPa/s. A normal tympanogram (type A) was defined as a peaked curve with compliance of 0.4 to $1.75 \mathrm{cc}$ and tympanic peak pressure (TPP) between -60 and +100 daPa. $^{3}$ Any curve different from the type A tympanogram was taken as abnormal. The positive pressure equalization test (PPET) was used in patients with perforated tympanic membranes. The air pressure in the canal was increased to $+200 \mathrm{daPa}$, and then the patients were asked to swallow 4 to 5 times within the period of 45 seconds to ascertain whether the positive pressure was being neutralized by each swallow. The pressure changes during swallowing were graphically recorded by the machine. The test was interpreted as follows - (a) normal ET function: positive pressure neutralizes completely from +200 daPa to between 0-50daPa; (b) partially impaired ET function: residual pressure of more than +50 daPa persists even after 4 to 5 swallows; (c) impaired ET function: positive pressure does not neutralize at all.

All patients had a nasal endoscopy with $0^{\circ}$ and $45^{\circ}$ rigid nasal endoscope to assess the pharyngeal orifice of the ET. The patients were asked to swallow to induce normal physiological tubal dilatations and to perform forced yawning to cause sustained tubal dilatation. The movements of the medial cartilaginous lamina, lateral excursion, and dilatory wave of the lateral wall and tubal lumen opening were studied. Eustachian tube movements were graded endoscopically based on the severity of the tubal pathology observed, as follows ${ }^{5}$ :

Grade 0: Normal ET with no mucosal edema or congestion. The medial cartilaginous lamina and lateral wall motions are normal. The tubal lumen opens well with swallowing.

Grade 1: Edema and congestion of mucosa limited to the pharyngeal orifice of the ET. Normal lateral wall motion, and the tubal lumen opens with swallowing.

Grade 2A: Reduced lateral wall motion secondary to edema and congestion involving the lumen. The tubal lumen opens partly with swallowing.

Grade 2B: Reduced lateral wall motion secondary to abnormal tubal muscle contraction. The tubal lumen opens partially with swallowing.

Grade 3A: The tubal lumen fails to open with swallowing secondary to gross edema.

Grade 3B: The tubal lumen fails to open with swallowing secondary to abnormal tubal muscle contraction.

All subjects with grade 0 ETs were assumed to have normal function, whereas grades 1, 2, and 3 and patulous ETs were assumed to be dysfunctional. McNemar's chisquared test was used to check the level of agreement between the two groups. 


\section{Results}

Of the total 106 patients, 54 were male and 52 were female. There were 72 patients with chronic suppurative otitis media (CSOM) - tubotympanic type, 12 with CSOM -atticoantral type, and 22 with adhesive otitis media.

Of the total 106 patients, 72 patients with CSOM -tubotympanic type had perforations in the pars tensa. A total of $33.3 \%$ of these patients showed abnormal findings on the PPET. Among the patients with adhesive otitis media, only three had type A tympanograms. This subgroup had the highest proportion of abnormal impedance (86.4\%). Patients with CSOM - atticoantral were found to have intact pars tensa and, therefore, were subjected to tympanometry. Only four patients had abnormal tympanometry curves. Of all the patients, 47 (44.3\%) had an abnormal finding on the impedance audiometry (-Table $\mathbf{1}$ ). There was a significant difference between the disease groups as estimated by the chi-squared test $(p<0.001)$.

Upon nasal endoscopy, a mere 9 out of the 106 patients (8\%) had normal findings. Overall, the most frequently observed tubal pathology was of grade 1 (45.3\%), followed by grade $2 \mathrm{~A}$ (17\%). In CSOM - tubotympanic type, the most frequently observed finding was of grade 1 in 34 patients (75\%). A similar pattern was also observed in patients with CSOM -atticoantral type too (75\%). However, in the cases of adhesive otitis media, the most common abnormality observed was of grade 2B (40.9\%), as depicted in - Table 2 .

Upon correlating the endoscopic grading with the impedance audiometry, we found that all the patients with grade 2B or higher nasal endoscopy had abnormal impedance audiometry findings. This indicates that the higher the grade, the higher the chance of an abnormal impedance audiometry (-Table 3).

Table 1 Impedance audiometry findings in patients with middle ear disease

\begin{tabular}{|l|l|l|l|}
\hline \multirow{2}{*}{ Disease } & \multicolumn{2}{|l|}{ Impedance audiometry } & \multirow{2}{*}{ Total } \\
\cline { 2 - 3 } & Normal & Abnormal & \\
\hline CSMO tubotympanic & 48 & 24 & 72 \\
\hline CSOM atticoantral & 8 & 4 & 12 \\
\hline Adhesive otitis media & 3 & 19 & 22 \\
\hline Total & 59 & 47 & 106 \\
\hline
\end{tabular}

Abbreviations: CSMO, chronic suppurative otitis media.
Table 3 Comparison between dynamic video endoscopy and impedance audiometry

\begin{tabular}{|l|l|l|l|}
\hline \multirow{2}{*}{$\begin{array}{l}\text { Nasal endoscopy } \\
\text { grading }\end{array}$} & \multicolumn{2}{|l|}{ Impedance audiometry } & \multirow{2}{*}{ Total } \\
\cline { 2 - 3 } & Normal & Abnormal & \\
\hline Grade 0 & 9 & 0 & 9 \\
\hline Grade 1 & 44 & 4 & 48 \\
\hline Grade 2A & 6 & 13 & 19 \\
\hline Grade 2B & 0 & 12 & 12 \\
\hline Grade 3A & 0 & 14 & 14 \\
\hline Grade 3B & 0 & 4 & 4 \\
\hline Total & 59 & 47 & 106 \\
\hline
\end{tabular}

The endoscopic grading of the 106 cases, when correlated with the middle ear manometry using McNemar's chisquared test, revealed that 56 cases showed complete agreement, and 50 cases showed disagreement (-Table 4). The $p$-value was 0.017 , which shows a significant correlation between the 2 tests. Upon correlating the endoscopic grading of the patients with CSOM tubotympanic type with the PPET using McNemar's chi-squared test, the observed agreement was of $48 \%$ (-Table 5 ). The $p$-value was 0.13 , which shows poor agreement between the 2 tests.

Table 4 Level of agreement between impedance audiometry and dynamic video endoscopy

\begin{tabular}{|l|l|l|}
\hline \multirow{2}{*}{} & \multicolumn{2}{|l|}{ Impedance audiometry } \\
\cline { 2 - 3 } & Abnormal & Normal \\
\hline Abnormal nasal endoscopy & 47 & 50 \\
\hline Normal nasal endoscopy & 0 & 9 \\
\hline
\end{tabular}

Table 5 Level of agreement between the PPET and dynamic video-endoscopy in patients with tubotympanic CSOM

\begin{tabular}{|l|l|l|}
\hline \multirow{2}{*}{} & \multicolumn{2}{|l|}{ PPET } \\
\cline { 2 - 3 } & Abnormal & Normal \\
\hline Abnormal nasal endoscopy & 24 & 39 \\
\hline Normal nasal endoscopy & 0 & 9 \\
\hline
\end{tabular}

Abbreviations: CSOM, chronic suppurative otitis media; PPET, positive pressure equalization test.

Table 2 Grading of Eustachian tube movement based on dynamic video endoscopy

\begin{tabular}{|c|c|c|c|c|c|c|c|}
\hline \multirow[b]{2}{*}{ Diseases } & \multicolumn{6}{|c|}{ Nasal endoscopy } & \multirow[t]{2}{*}{ Total } \\
\hline & Normal & Grade 1 & Grade 2A & Grade 2B & Grade 3A & Grade 3B & \\
\hline CSOM tubotympanic & 9 & 34 & 12 & 3 & 13 & 1 & 72 \\
\hline CSOM atticoantral & 0 & 9 & 3 & 0 & 0 & 0 & 12 \\
\hline Adhesive otitis media & 0 & 5 & 4 & 9 & 1 & 3 & 22 \\
\hline Total & 9 & 48 & 19 & 12 & 4 & 4 & 106 \\
\hline
\end{tabular}




\section{Discussion}

The etiology and pathology of middle ear diseases are multifactorial, and include infectious, allergic, immunologic, environmental and social factors. It is a well-known fact that ET dysfunction plays a very important role in the pathogenesis of middle ear diseases. ${ }^{6}$ As there is no single test which can assess both the anatomic and physiological functions of the ET, the present study was performed to assess the correlation of dynamic video-endoscopic grading of the ET movement with impedance audiometry in patients with middle ear diseases.

Impedance audiometry is a measure of the resistance offered by the tympanic membrane to sound energy striking it. This test is affected by the mass, mobility and resistance systems of the external and middle ear cavities. Our study showed that the tympanometry findings were dependent on the type of middle ear disease ( $\mathbf{- T a b l e} \mathbf{1}$ ).

Dynamic video endoscopy aids in the visualization and assessment of the movements of the nasopharyngeal orifice of the ET, which is a useful technique in diagnosing the underlying pathologic conditions in dysfunctional tubes. Grading of the ET function would play an important role in predicting the prognosis after surgery for middle ear diseases. In the present study, the failure of the tube to dilate was most commonly caused by mucosal edema and congestion of the nasopharyngeal orifice (49.5\%), followed by reduced lateral wall motion secondary to the edema and congestion involving the lumen, which causes the tubal lumen to partially open while swallowing ( - Table 2). These findings are in accordance with a study by Poe et al ${ }^{7}$ in which they analyzed dysfunctional ETs by video endoscopy on 64 ears with middle ear diseases and noted mucosal edema with reduced lateral wall motion as the most common cause of tubal dysfunction. ${ }^{8}$ Poe et al did not consider a tube dysfunctional if the edema was limited to its nasopharyngeal orifice, but in the present study, such tubes were classified as having grade1 tubal dysfunction, since edema and congestion represent an inflammatory process that could progress and later lead to tubal dysfunction.

Eustachian tube dysfunction by mucosal edema could be caused by bulk, increased wall stiffness; reduced tensor veli palatini efforts; or both. In the present study, 16 Eustachian tubes (15.1\%) had dysfunctions secondary to abnormal tubal muscle contraction ( $\mathbf{- T a b l e ~} \mathbf{2}$ ). This could be related to the action of the tensor veli palatini, which is the active dilator of the ET. The abnormal muscle action could be due to primary muscle weakness, inadequate insertion of the tensor veli palatini or the dilator tubae, or the lack of coordinated effort between the tensor veli palatini and the dilator tubae. In our study, all cases of adhesive otitis media showed ET dysfunction (-Table 2), proving once again that ET dysfunction is the main contributory factor in the pathogenesis of adhesive otitis media.

Upon correlating the endoscopy findings with the impedance audiometry, 56 cases showed complete agreement, and 50 cases showed disagreement in the present study (-Table 4). The patients with grade 2 A or higher of dysfunction showed abnormal impedance audiometry. These findings are in agreement with previously published results that emphasized a significant correlation between the two tests. ${ }^{5,8,9}$ The results of the present study show that as the severity of the ET dysfunction increases, so does the chance of occurrence of middle ear pressure dysfunction. This, however, also means that many patients with minimal ET dysfunction may have normal middle ear pressure. In - Table 3 it is clearly demonstrated that out of the 48 patients with grade 1 tubal dysfunction, only 4 (8.3\%) patients had abnormal impedance function, while out of the 16 patients with grade $2 \mathrm{~A}$ tubal dysfunction, 13 (68.5\%) patients had abnormal impedance function, and all the patients with grades $2 \mathrm{~B}$ and 3 had abnormal middle ear pressure. These results are of practical significance for the diagnosis and treatment of middle ear diseases.

Patients with middle ear diseases like CSOM with severe ET dysfunction are likely to have middle ear pressure abnormalities, and surgery may have poorer prognosis when compared with patients with minimal ET dysfunction. Hence, patients can be counselled about prognosis following surgery. This also means that the correction of the ET abnormality is of prime importance in the management of middle ear diseases by adenoidectomy, for example. It is, however, important to note that in this study all patients with CSOM Atticoantral disease had mild ET dysfunctions (grades 1 and 2A). In the cases of tubotympanic disease, the proportion of patients with mild ET dysfunction was $70 \%$, and this means that it is likely that ET dysfunction contributed more to the development of tubotympanic disease than to the development of atticoantral CSOM. This reinforces the need to treat a focus of infection in the paranasal sinuses or tonsils in all patients with tubotympanic disease as these localized infections contribute significantly to ET dysfunction.

In the present study, $91.5 \%$ of the cases of ET dysfunction were identified by nasal endoscopy, while impedance audiometry showed only $44.3 \%$ of the cases with abnormal findings ( - Table 3).Therefore, when assessing the correlation between the ET dysfunction and the abnormal impedance audiogram, it will appear that in almost $50 \%$ of the cases there is no correlation. However, this can be explained by the fact that the majority of cases of middle ear disease (57\%) have grade 0 or grade 1 tubal abnormalities. This means that even minor ET dysfunctions can contribute to middle ear disease, but most of the patients with mild ET dysfunction will have no middle ear pressure anomaly. This can be explained by the fact the ET abnormality, especially edema, might have been severe at the onset of the disease, but as the edema settled, it left a sequel, namely a tympanic membrane perforation that has not healed due to factors unrelated to middle ear pressure abnormalities. This could also explain the poor agreement between the PPET and the dynamic video endoscopy. It is interesting to note that in all patients with glue ear there was an ET dysfunction; this means that the persistent disease of the ET has led to a persistent middle ear pressure anomaly, and that the condition would improve only if the ET dysfunction is corrected.

A study similar to the present one, by Mathew et al, showed a significant correlation between dynamic ET endoscopy and middle ear manometry. The most common cause of tubal dysfunction in their study was mucosal edema 
followed by uncoordinated or absent lateral wall movement. ${ }^{5}$ About $91.5 \%$ of the cases in the present study also showed ET dysfunction that had mucosal edema as the main cause ( - Table 2 ). The consonance of the results between the study by Mathew et al and ours might be due to the same ethnic patient population involved.

In similar studies by Augustine et al and Chauhan et al on the efficacy of dynamic slow motion video endoscopy as a test of ET function, a statistically significant correlation between dynamic slow motion video endoscopy and the Eustachian swallow test was observed. ${ }^{8,9}$ They concluded that nasal endoscopy appeared to overdiagnose ET dysfunction when used alone.

In a pilot study conducted by Handel et al about the synchronous endoscopy and sonotubometry of the ET, it was concluded that simultaneous synchronous endoscopy and sonotubometry may improve the efficacy of either performed separately as a tool for measurement of ET function. ${ }^{10}$ Although there are several manometric tests for the indirect assessment of tubal function, such as tympanometry, the forced opening test, the inflation-deflation test, and sniff tests, Bunne et al concluded that a single test of the ET function is of low prognostic value. ${ }^{11}$ All direct tests, such as mucociliary clearance tests, computed tomographic studies, and radiographic studies are time consuming, cumbersome, or expensive.

\section{Conclusion}

A significant alteration in middle ear pressure was observed in patients with severe ET dysfunction (grades 2A, 2B, 3A, 3B assessed by endoscopy). However, there was no significant correlation between the nasal endoscopy findings and the impedance audiometry results in the cases of mild (grade 1) ET dysfunction, which was what most patients had. Since impedance audiometry fails to detect minor ET dysfunction, it may be coupled with other techniques, like nasal endoscopy, to improve the sensitivity of the ET function assessment. Further research needs to be performed to find out if grade 1 ET dysfunction is of any clinical significance in the treatment outcome of middle ear diseases.

\section{Conflicts of Interest}

The authors have no conflict of interest to declare.

\section{Ethical Approval}

All procedures involving human participants were in accordance with the ethical standards of the institutional and/or national research committee, and with the 1964 Helsinki declaration and its later amendments or comparable ethical standards. Informed consent was obtained from all individual participants included in the study.

\section{References}

1 Pau HW. [Eustachian tube and middle ear mechanics]. HNO 2011; 59(10):953-963

2 Sato H, Nakamura H, Honjo I, Hayashi M. Eustachian tube function in tympanoplasty. Acta Otolaryngol Suppl 1990;471:9-12

3 Smith ME, Tysome JR. Tests of Eustachian tube function: a review. Clin Otolaryngol 2015;40(04):300-311

4 Poe DS, Pyykkö I. Measurements of Eustachian tube dilation by video endoscopy. Otol Neurotol 2011;32(05):794-798

5 Mathew GA, Kuruvilla G, Job A. Dynamic slow motion video endoscopy in eustachian tube assessment. Am J Otolaryngol 2007;28(02):91-97

6 Grimmer JF, Poe DS. Update on eustachian tube dysfunction and the patulous eustachian tube. Curr Opin Otolaryngol Head Neck Surg 2005;13(05):277-282

7 Poe DS, Abou-Halawa A, Abdel-Razek O. Analysis of the dysfunctional eustachian tube by video endoscopy. Otol Neurotol 2001;22 (05):590-595

8 Augustine AM, Varghese L, Michael RC, Albert RR, Job A. The efficacy of dynamic slow motion video endoscopy as a test of eustachian tube function. J Laryngol Otol 2013;127(07): 650-655

9 Chauhan B, Chauhan K. A comparative study of eustachian tube functions in normal and diseased ears with tympanometry and videonasopharyngoscopy. Indian J Otolaryngol Head Neck Surg 2013;65(Suppl 3):468-476

10 Handzel O, Poe D, Marchbanks RJ. Synchronous endoscopy and sonotubometry of the eustachian tube: a pilot study. Otol Neurotol 2012;33(02):184-191

11 Bunne M, Falk B, Magnuson B, Hellström S. Variability of Eustachian tube function: comparison of ears with retraction disease and normal middle ears. Laryngoscope 2000;110(08):1389-1395 